\title{
A Rare Case of Lymph Node Metastasis from Early Gastric Cancer
}

\author{
Takaaki Yoshikawa ${ }^{1}$, Yoshio Kadokawa ${ }^{2}$, Masaya Ohana ${ }^{3}$, Akihisa Fukuda ${ }^{1}$ and Hiroshi Seno ${ }^{1}$ \\ ${ }^{1}$ Department of Gastroenterology and Hepatology, Kyoto University Graduate School of Medicine, Kyoto, Department of ${ }^{2}$ Gastrointestinal \\ Surgery, ${ }^{3}$ Gastroenterology, Tenri Hospital, Tenri, Nara, Japan
}

Gastric cancers that fulfill the Japanese criteria for curative endoscopic resection show a low risk of lymph node (LN) metastasis. Here, we report a case of LN metastasis from early gastric cancer that fulfilled the curative criteria. A 74-year-old Japanese woman was referred to our hospital for treatment of early gastric cancer identified at the site of a hyperplastic polyp that had been diagnosed 10 years prior to presentation. Contrast-enhanced computed tomography did not show any lymphadenopathy and laparoscopy-assisted distal gastrectomy was performed. Histopathological examination revealed a predominantly moderately differentiated adenocarcinoma that measured $15 \mathrm{~mm}$ in size and was confined to the mucosa. However, a single metastatic regional LN was observed. A few cancer cells showed positive staining for alpha-fetoprotein. It should be noted that early gastric cancer can be accompanied by LN metastasis even if it fulfills the criteria for curative endoscopic resection. Clin Endosc 2019;52:369-372

Key Words: Early gastric cancer; Lymph node metastasis; Alpha-fetoproteins; Gastric hyperplastic polyp; Endoscopic resection

\section{INTRODUCTION}

Recently, endoscopic submucosal dissection (ESD) and endoscopic mucosal resection (EMR) have been widely accepted as less invasive and safe treatment methods for early gastric cancer (EGC). The guidelines published by the Japan Gastroenterological Endoscopy Society in 2016 define the curative resection criteria for ESD and EMR. ${ }^{1}$ According to the guidelines, en bloc resection of a lesion measuring $<2 \mathrm{~cm}$ in diameter, predominantly of the differentiated type, pTla, UL (-), ly $(-), \mathrm{v}(-)$ is considered a curative resection. Here, we report a case of EGC with lymph node (LN) metastasis that fulfilled the criteria for curative endoscopic resection. Histopathological examination of the EGC revealed a predominantly moderately differentiated adenocarcinoma with a small poorly

Received: July 26, 2018 Revised: September 7, 2018

Accepted: September 10, 2018

Correspondence: Takaaki Yoshikawa

Department of Gastroenterology and Hepatology, Kyoto University Graduate School of Medicine, 54 Kawahara-cho, Syogoin, Sakyo-ku, Kyoto 606-8507, Japan Tel: +81-75-751-4319, Fax: +81-75-751-4303, E-mail: anctan180@gmail.com ORCID: https://orcid.org/0000-0002-3173-4360

cc This is an Open Access article distributed under the terms of the Creative Commons Attribution Non-Commercial License (http://creativecommons.org/ licenses/by-nc/3.0) which permits unrestricted non-commercial use, distribution, and reproduction in any medium, provided the original work is properly cited. differentiated component. The lesion had originated from a gastric hyperplastic polyp (HP) and contained alpha-fetoprotein (AFP)-producing cancer cells.

\section{CASE REPORT}

A 74-year-old woman was referred to our hospital for treatment of EGC. She had been diagnosed with an HP 10 years prior to presentation (Fig. 1A). The diagnosis of the HP (located on the posterior wall of the antrum) was confirmed using histopathological examination (Fig. 1B). After the HP was detected, annual esophagogastroduodenoscopic examinations were performed at our hospital until 4 years prior. During this period, the polyp remained unchanged in shape and size.

Presently, esophagogastroduodenoscopy revealed a type 0 -I adenocarcinoma at the site of the previous HP (Fig. 2A). The lesion measured approximately $20 \mathrm{~mm}$ in size without an ulcer and appeared to be confined to the mucosal layer. A biopsy specimen of the lesion revealed a mixture of well-differentiated and poorly differentiated adenocarcinoma. Contrast-enhanced computed tomography (CT) did not reveal any LN enlargement or distant metastasis (Fig. 2B). Routine hematological examination and biochemical tests were within 
normal limits. AFP was also within normal limits at $3.8 \mathrm{ng} /$ $\mathrm{mL}$ (normal range $[\mathrm{NR}]<10 \mathrm{ng} / \mathrm{mL}$ ). Helicobacter pylori-specific immunoglobulin $\mathrm{G}$ antibodies in the serum were negligble at $6 \mathrm{U} / \mathrm{mL}(\mathrm{NR}<10 \mathrm{U} / \mathrm{mL})$. Her EGC showed an absolute indication for endoscopic resection; however, she decided to undergo surgery. Laparoscopy-assisted distal gastrectomy with D1 lymphadenectomy (including LN No. 1, 3, 4sb, 4d, 5, 6, 7, 8a, 9) was performed.

Histopathological examination of the resected specimen revealed a type 0 -I lesion measuring $15 \mathrm{~mm}$ in size (Fig. 3A). The specimen predominantly showed a moderately differentiated adenocarcinoma (Fig. 3B) with a small poorly differentiated component (Fig. 3C). In addition to routine histopathological analysis that evaluated tissue sections no more than 5 $\mathrm{mm}$ of thickness, the resected specimen was examined with a deeper cut. The EGC was confined to the mucosal layer, and no lymphatic and vascular invasion were observed based on immunostaining using D2-40 and CD31 stains, respectively. Nearly the entire HP was replaced by the adenocarcinoma; however, a small residual segment of hyperplastic epithelium was observed (Fig. 3D). A few cancer cells showed positive AFP staining (Fig. 3E). Most tumor cells were aligned to form a tubular pattern and showed clear cytoplasm. Moreover, this tumor did not contain a hepatoid component in which cancer cells with eosinophilic cytoplasm are arranged in a solid and trabecular pattern. Therefore, we diagnosed the tumor as an adenocarcinoma with enteroblastic differentiation rather than a hepatoid adenocarcinoma and considered this to be a variant of AFP-producing gastric cancers (AGCs).

Surprisingly, histopathological examination revealed metas-
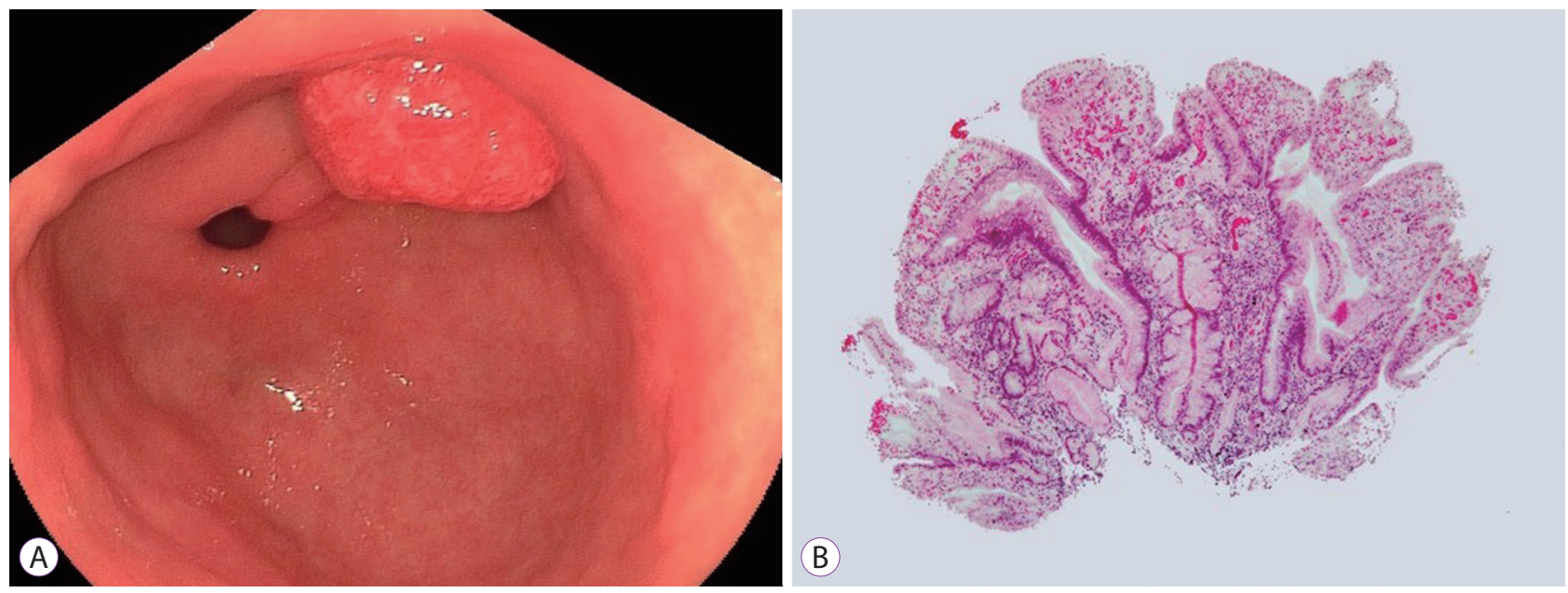

Fig. 1. Image shows a gastric hyperplastic polyp identified 10 years prior to the initiation of treatment. (A) Esophagogastroduodenoscopic image shows a reddish pedunculated polyp on the posterior antral wall of the stomach. (B) The biopsy specimen obtained from the polyp shows hyperplastic foveolar epithelium (hematoxylin and eosin, $\times 4)$.
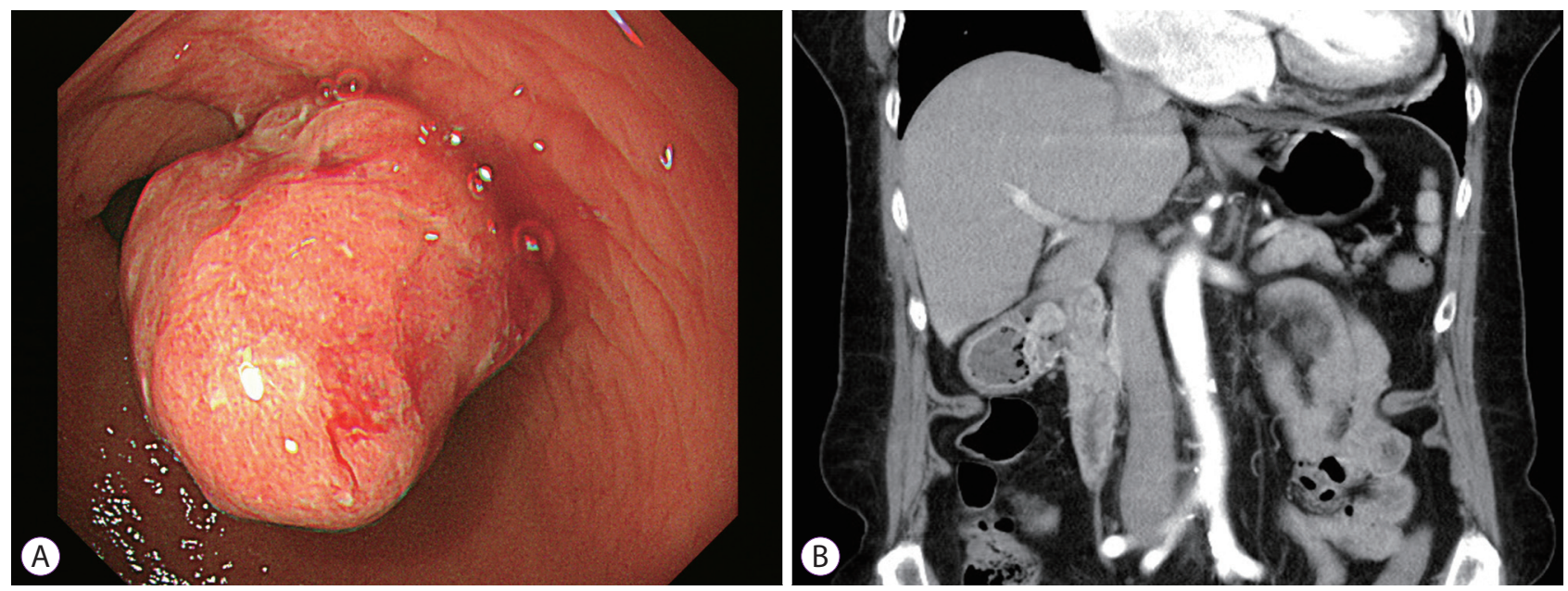

Fig. 2. Images show early gastric cancer observed at the time of treatment. (A) An esophagogastroduodenoscopic image shows a type 0-I adenocarcinoma at the site of the previously identified hyperplastic polyp. (B) A contrast-enhanced computed tomographic image does not show any lymph node enlargement. 
tasis in a single No. 6 group LN (Fig. 4A, B). The LN metastasis was composed of a moderately differentiated adenocarcinoma and was negative for AFP expression.

The patient did not receive additional chemotherapy and has been followed-up for 2 years without recurrence.

\section{DISCUSSION}

We present a case of LN metastasis from a small, predominantly moderately differentiated EGC that was confined to the mucosal layer without ulcerative findings or lymphovascular invasion and fulfilled the criteria for curative endoscopic resection based on Japanese gastric cancer treatment guidelines. ${ }^{1}$

A previous study has shown that LN metastasis was observed in 0 of 274 surgical specimens that were of differentiated type (well- and moderately differentiated tubular adenocarcinoma), measured $\leq 20 \mathrm{~mm}$ in size, and limited to the mucosal layer without ulcerative findings. ${ }^{2}$ Based on the latest Japanese guidelines, a lesion resected en bloc, measuring $<2 \mathrm{~cm}$ in diameter, predominantly of the differentiated type, pTla, UL (-), ly (-), v (-), with negative surgical margins is considered a curative resection. ${ }^{1}$ EGC with LN metastasis after curative resection by ESD has been reported in 2 previous cas-
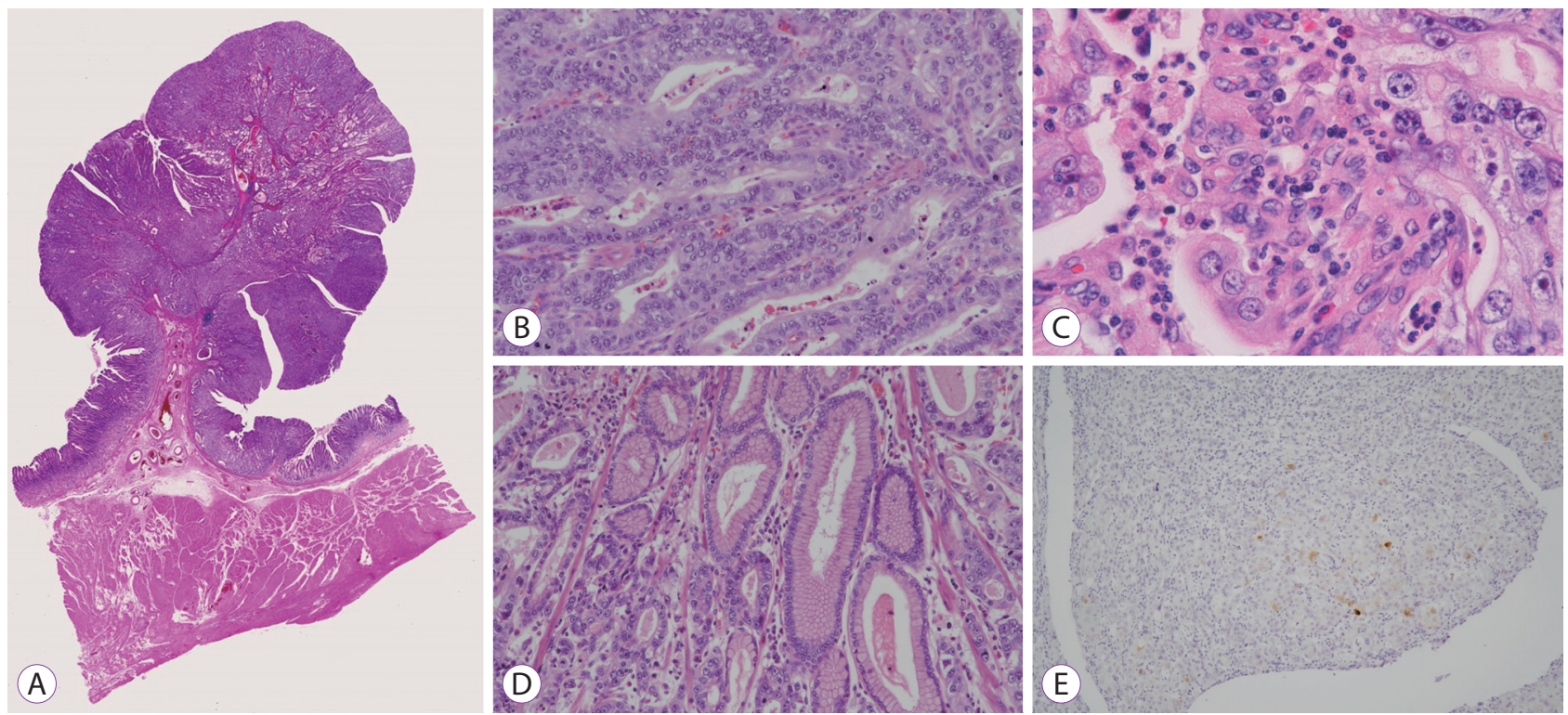

Fig. 3. Histopathological findings of the primary lesion. (A) The resected specimen shows a type 0 -I adenocarcinoma measuring $15 \mathrm{~mm}$ in size that is confined to the mucosal layer (hematoxylin and eosin $[\mathrm{H \& E}], \times 1)$ ). (B) The specimen predominantly shows a moderately differentiated adenocarcinoma (H\&E, $\times 200)$. (C) The specimen shows a small poorly differentiated component (H\&E, $\times 400)$. (D) The specimen shows a small segment of residual hyperplastic epithelium (H\&E, $\times 200)$. ( $E$ ) Immunohistochemical examination shows a few cancer cells stained positively for alpha-fetoprotein (H\&E, $\times 100)$.
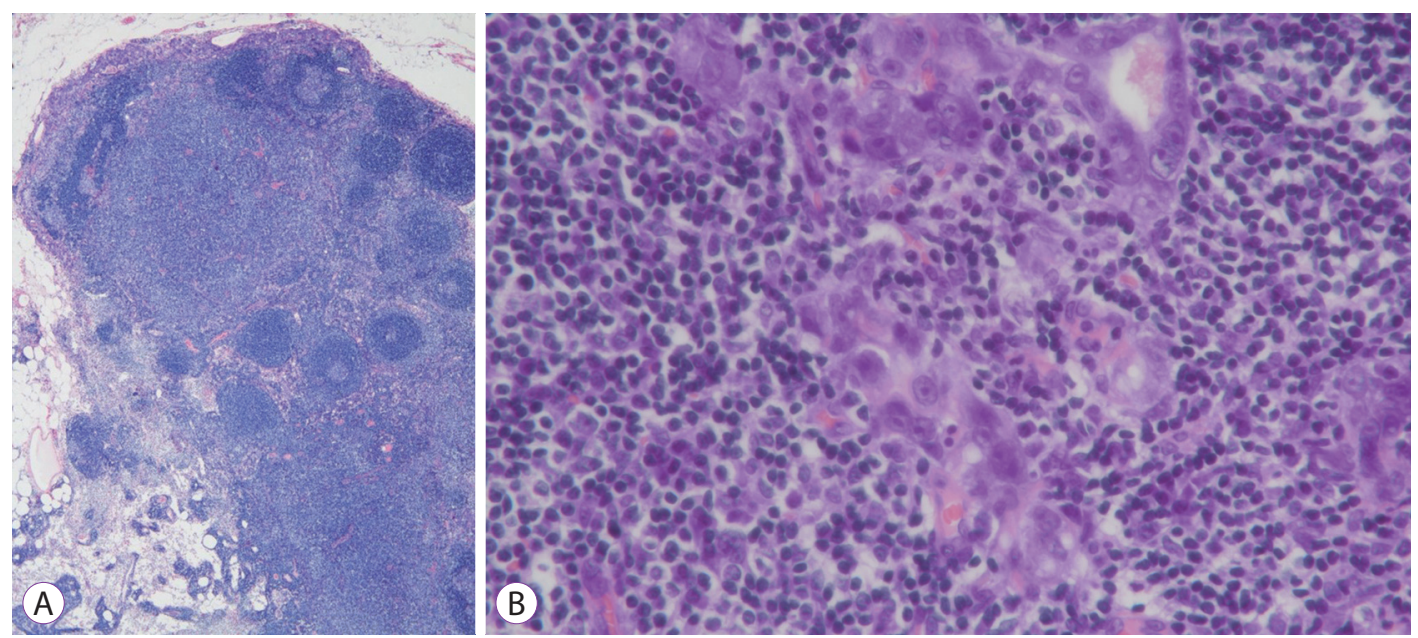

Fig. 4. Image shows a metastatic lymph node with features of a moderately differentiated adenocarcinoma (hematoxylin and eosin, $A \times 40$ and $B \times 400$ ). 
es. ${ }^{3,4}$ However, in both cases, LN enlargement was detected before the ESD was performed. In contrast, no LN enlargement was identified preoperatively in our patient. Therefore, if ESD had been performed, the lesion would have been considered a curative resection without requiring additional surgery.

It should be noted that the tumor contained AGC cells in this case. A previous study has shown that the following features in AGCs were significantly more prominent than those in ordinary gastric cancers: occurrence of $\mathrm{LN}$ metastasis $(96 \%$ vs. $50 \%$ ), venous invasion ( $83 \%$ vs. $47 \%$ ), liver metastasis $(72 \%$ vs. $10 \%$ ), and low 5 -year survival rates (12\% vs. $53 \%){ }^{5}$ This aggressive behavior of AGCs might cause LN metastasis during the early stages, as was observed in our patient. Adenocarcinoma with enteroblastic differentiation (occurring as a variant of AGCs) is associated with a high incidence of LN metastasis, liver metastasis, and poor prognosis. ${ }^{6}$ Recently, AFP producing EGCs are being detected more frequently in clinical practice. These lesions also tend to show a poor prognosis and a high incidence of LN metastasis with liver involvement. ${ }^{\text {? }}$

Generally, the risk of LN metastasis in poorly differentiated gastric adenocarcinoma and signet ring cell gastric carcinoma is higher than that in well- and moderately differentiated gastric adenocarcinoma. ${ }^{2}$ Moreover, reportedly, the mixed histological type, containing differentiated and undifferentiated components demonstrates a high risk of LN metastasis. ${ }^{8}$ Although our patient showed the mixed histological type, it is unknown whether the histological features affected LN metastasis because the poorly differentiated component constituted only a small part of the primary lesion and because most of the LN metastasis tissue comprised moderately and not poorly differentiated components.

In our case, the EGC originated in an HP. HPs are generally considered benign lesions, although they may show malignant potential. Nakamura et al. ${ }^{9}$ proposed the following histopathological criteria that suggest malignant transformation: (1) coexistence of benign and malignant lesions in the same polyp, (2) sufficient evidence to indicate the malignant lesion had previously been a benign polyp and, (3) sufficient cellular and structural atypia in the malignant lesion. In our case, the tumor satisfied these criteria. However, the association between HP-associated EGC and LN metastasis is unclear.

Several endoscopic treatments are presently available for EGC owing to advances in endoscopic skills and knowledge. ESD and EMR should be performed when the likelihood of LN metastasis is extremely low. Imaging studies such as contrast-enhanced CT and endoscopic ultrasonography should be performed for assessment of LN metastasis or distant metastasis before endoscopic treatment. Notably, the size of LNs is not a reliable indicator of LN metasitasis. ${ }^{10}$ It is difficult to detect small LN metastasis and inflammatory LNs are often indistinguishable from metastatic LNs. Because imaging studies show limitations, detailed histopathological examination of the endoscopically resected primary lesion is necessary. A greater number of cases of EGC with LN metastasis need to be studied to gain a better understanding of the tumor to devise an optimal treatment strategy.

\section{Conflicts of Interest}

The authors have no financial conflicts of interest.

\section{Author Contributions}

Resources: Takaaki Yoshikawa, Yoshio Kadokawa, Masaya Ohana Supervision: MO, Akihisa Fukuda, Hiroshi Seno

Visualization: TY

Writing-original draft: TY

Writing-review\&editing: MO, AF, HS

\section{REFERENCES}

1. Ono H, Yao K, Fujishiro M, et al. Guidelines for endoscopic submucosal dissection and endoscopic mucosal resection for early gastric cancer. Dig Endosc 2016;28:3-15.

2. Gotoda T, Yanagisawa A, Sasako M, et al. Incidence of lymph node metastasis from early gastric cancer: estimation with a large number of cases at two large centers. Gastric Cancer 2000;3:219-225.

3. Kim DJ, Kim W. A case of single lymph node metastasis near the common hepatic artery following a curative endoscopic resection for gastric mucosal cancer. Gastric Cancer 2014;17:387-391.

4. Hasegawa F, Kiyozaki H, Takata O, et al. Lymphatic invasion in small differentiated-type mucosal gastric cancer. Clin J Gastroenterol 2012;5:234-238

5. Chang YC, Nagasue N, Abe S, Taniura H, Kumar DD, Nakamura T. Comparison between the clinicopathologic features of AFP-positive and AFP-negative gastric cancers. Am J Gastroenterol 1992;87:321-325.

6. Murakami T, Yao T, Mitomi H, et al. Clinicopathologic and immunohistochemical characteristics of gastric adenocarcinoma with enteroblastic differentiation: a study of 29 cases. Gastric Cancer 2016;19:498-507.

7. Tazaki S, Yoshida H. [A case of AFP-producing early gastric cancer that grew in the short-term]. Progress of Digestive Endoscopy 2015;87:116117.

8. Hanaoka N, Tanabe S, Mikami T, Okayasu I, Saigenji K. Mixed-histologic-type submucosal invasive gastric cancer as a risk factor for lymph node metastasis: feasibility of endoscopic submucosal dissection. Endoscopy 2009;41:427-432.

9. Nakamura T, Nakano G. Histopathological classification and malignant change in gastric polyps. J Clin Pathol 1985;38:754-764.

10. Mönig SP, Zirbes TK, Schröder W, et al. Staging of gastric cancer: correlation of lymph node size and metastatic infiltration. AJR Am J Roentgenol 1999;173:365-367. 